\title{
Investigation on dynamics of red blood cells through their behavior as biophotonic lenses
}

\author{
Pasquale Memmolo \\ Francesco Merola \\ Lisa Miccio \\ Martina Mugnano \\ Pietro Ferraro
}




\title{
Investigation on dynamics of red blood cells through their behavior as biophotonic lenses
}

\author{
Pasquale Memmolo, ${ }^{*}$ Francesco Merola, Lisa Miccio, Martina Mugnano, and Pietro Ferraro \\ National Council of Research-Istituto di Scienze Applicate e Sistemi Intelligenti “E. Caianiello," Via Campi Flegrei 34, 80078 Pozzuoli, Naples, Italy
}

\begin{abstract}
The possibility to adopt biological matter as photonic optical elements can open scenarios in biophotonics research. Recently, it has been demonstrated that a red blood cell (RBC) can be seen as an optofluidic microlens by showing its imaging capability as well as its focal tunability. Moreover, correlation between an RBC's morphology and its behavior as a refractive optical element has been established and its exploitation for biomedical diagnostic purposes has been foreseen. In fact, any deviation from the healthy RBC morphology can be seen as additional aberration in the optical wavefront passing through the cell. By this concept, accurate localization of focal spots of RBCs can become very useful in the blood disorders identification. We investigate the three-dimensional positioning of such focal spots over time for samples with two different osmolarity conditions, i.e., when they assume discocyte and spherical shapes, respectively. We also demonstrate that a temporal variation of an RBC's focal points along the optical axis is correlated to the temporal fluctuations in the RBC's thickness maps. Furthermore, we show a sort of synchronization of the whole erythrocytes ensemble. (C) 2016 Society of Photo-Optical Instrumentation Engineers (SPIE) [DOI: 10.1117/1.JBO.21.12.121509]
\end{abstract}

Keywords: wavefront analysis; biophotonics lenses; digital holography; microscopy.

Paper 160402SSRR received Jun. 13, 2016; accepted for publication Sep. 19, 2016; published online Oct. $13,2016$.

\section{Introduction}

Frontiers in biophotonics could be opened by adopting biological matter as an optical element. ${ }^{1-3}$ Recently, it has been discovered that a red blood cell (RBC) behaves as a tunable optofluidic biophotonic lens. ${ }^{1}$ Healthy erythrocytes have typically flexible biconcave disk-like shapes with homogeneous distribution of the refractive index. Pathological conditions affecting erythrocytes can significantly modify their natural discocyte shape, thus full morphologic characterization of RBCs is relevant for diagnostics. In fact, blood disorders are typically reflected in specific findings from erythrocytes analysis and their morphometric signature can furnish a complete diagnosis as demonstrated by challenging recent results. ${ }^{4-6}$ Several studies focus the attention on the imaging technique used to recover the morphology of RBCs for diagnostic purposes..$^{7-9}$ In this sense, digital holography (DH) in microscopy ${ }^{10-13}$ has proved to be a very powerful label-free, noninvasive technique for quantitative phase imaging (QPI) of erythrocytes. ${ }^{14-28}$ In particular, in Ref. 15, the three-dimensional (3-D) morphometric analysis of flowing and not-adherent RBCs was carried out by using a shape from a silhouette algorithm. ${ }^{29}$ In this case, a simple holographic approach, in combination with holographic optical tweezers, has been demonstrated for accurate calculation of RBCs biovolume, displaying their 3-D shapes, and classifying them in terms of morphology. QPI by DH has been also applied by Moon et al. ${ }^{16}$ for automated tracking of temporal displacements of RBCs and analysis of 3-D morphology of RBCs stored in different periods. ${ }^{18}$ Moreover, blood disease identification through QPI methods have been explored for 3-D morphometry of erythrocytes measuring volumes, refractive indices, and morphological parameters. ${ }^{1,8}$ However, all the aforementioned

*Address all correspondence to: Pasquale Memmolo, E-mail: p.memmolo@ isasi.cnr.it methods require quite complex image processing for morphology retrieval in three-dimensional and 3-D and, most important, the diagnosis is subjective, as it depends on the physician's personal evaluation of QPI images.

Recently, it has been demonstrated that erythrocytes can be seen as an optofluidic biolenses ${ }^{1}$ by demonstrating their focus tunability, imaging capability, and allowing the investigation of their 3-D morphology through wavefront analysis by using Zernike polynomials. Consequently, a healthy RBC can be modeled as a biolens having two main aberrations, i.e., focus shift and third-order spherical aberration, respectively. Furthermore, due to its typical central convex shape, an erythrocyte provides two focal planes, identified by a real and a virtual focal loci. Any deviations from the biconcave disk-like shape produce a change in such foci. In fact, in analogy to adaptive optics testing, blood diagnosis has been demonstrated by screening abnormal cells through wavefront analysis applied to RBCs ensemble as a biolens array. However, it is well known that membranes of living RBCs fluctuate continuously. Consequently, it is important to study if, and how much, the mentioned fluctuations affect the foci positions in order to understand if a diagnostic based on focal spot analysis is impaired by such dynamic behavior. It is important to note that temporal fluctuations of the focal points loci have not been investigated yet, despite many studies in literature on membrane fluctuations.

In fact, investigations about blood membranes fluctuation phenomenon are widely reported, ${ }^{24,30-34}$ where the strong correlation between membrane dynamics and the characterization of blood-related diseases is demonstrated. ${ }^{24}$ Since erythrocytes membrane fluctuations also affect the calculation of the RBC biolens aberrations, the position of the focus planes along the optical axis changes. Here, we report comprehensive

$1083-3668 / 2016 / \$ 25.00$ @ 2016 SPIE 

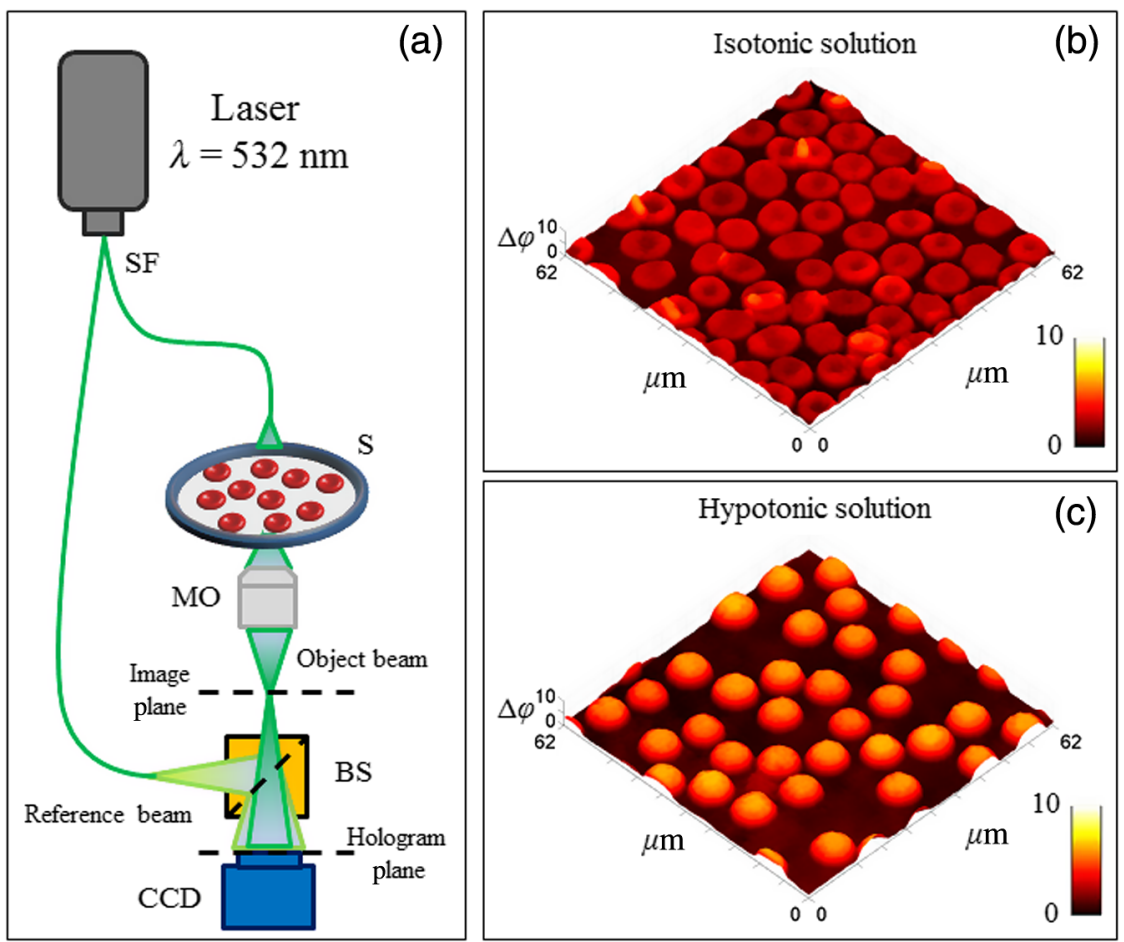

Fig. 1 (a) Optical setup for RBCs holographic recording. (b) and (c) Phase map reconstructions of discocytes ensemble and spherical-like erythrocytes ensemble, respectively.

measurement of focal spots stability of an RBCs ensemble in different experimental conditions. This study is preliminary but it opens a way for the detection of blood diseases through focal spots analysis. Specifically, we implement a 3-D tracking algorithm $^{35,36}$ to locate, along the optical axis, both real and virtual foci fluctuation over time. We tested two samples with different osmolarity conditions, i.e., isotonic solution, where healthy RBCs have the classical discocyte shape, and the hypotonic solution, where erythrocytes present sphericallike shapes, respectively. In addition, we reveal that (i) focus loci stability in 3-D is influenced by the intensities of temporal fluctuations in the RBC's thickness map, thus, a low fluctuation of the RBC membrane corresponds to a relative small variation of the position of its foci along the optical axis and vice versa and (ii) the positions of focal spots along the optical axis are synchronized, with an average movements' correlation greater than 0.5 in both experiments.

\section{Materials and Methods}

\subsection{Experimental Setup and Samples Preparation}

The experiments are realized with heparinized blood drawn within the hour before use. Blood is withdrawn into a hematocrit tube and centrifuged at room temperature, for $15 \mathrm{~min}$ at 2500 revolutions per minute, in order to obtain separated component parts as plasma, buffy coat, and RBCs at the bottom of the centrifuge tube. We consider two different osmolarities of the medium, i.e., $\sim 308 \mathrm{mOsm} / \mathrm{L}$ that is isotonic with the membrane of RBCs where healthy erythrocytes appear as classical biconcave disk-like shapes, and a hypotonic solution at $\sim 205 \mathrm{mOsm} / \mathrm{L}$, where RBCs assume a spherical-like shape. The two samples are imaged by using a classical $\mathrm{DH}$ in microscopy setup, as sketched in Fig. 1(a). The laser light source is coupled into an optical fiber, which splits the laser light into an object beam (OB) and reference beam. The OB impinges on the sample (S) and passes through a $100 \times$ microscope object before both beams are recombined using a beam splitter and are collected by the $1024 \times 1024$ CCD camera at 10 frames per seconds. Specifically, we recorded two holographic time sequences (5 min each one, about 500 frame acquired) of the RBCs ensemble for both discocytes and spherical-like erythrocytes, with the aim to inspect their self-motility. Figures 1(b) and 1(c) show the first QPI reconstructions of time sequences of both samples. Notice that the RBCs ensemble is placed on the bottom surface of the chamber and no transversal migration occurs, a part of the Brownian motion (with null average value) around their centers of mass.

\subsection{Red Blood Cells Wavefront Analysis: Focal Spots Detection and Tracking}

To investigate the focal spots' stability of erythrocytes ensemble in time, we implemented the following steps:

i. Phase maps segmentation, by using the anisotropic diffusion filter, ${ }^{37}$ with the aim to extract isolated $\mathrm{RBC}$ of each QPI reconstruction, i.e., removing superposed cells.

ii. Numerical propagation of each segmented phase reconstruction along the optical axis. In the case of discocytes, intensity distributions in the transverse planes and along the optical axis (plane $X-Z$ and plane $Y-Z$ ) are used to detect both virtual and real foci, while for spherical-like erythrocytes only real foci are detected.

iii. 3-D tracking of foci, by identification their reconstruction planes through refocusing criterion based on the 

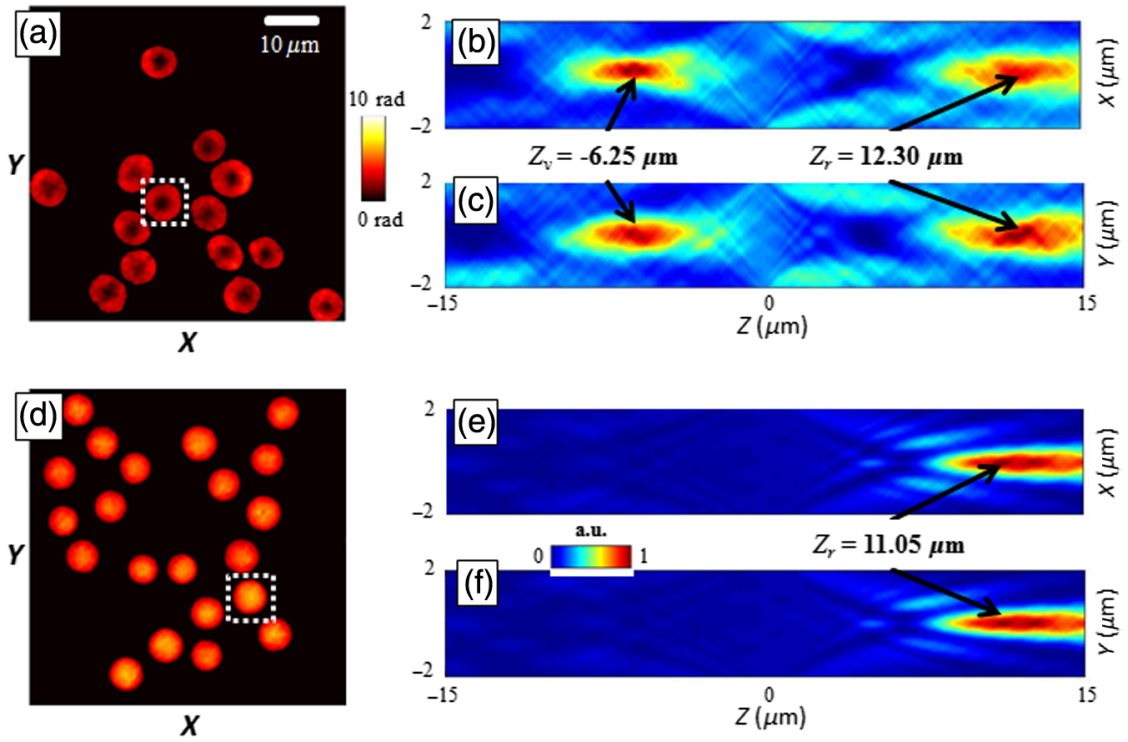

Fig. 2 Segmented QPI reconstructions, obtained after the anisotropic diffusion filtering, for (a) discocytes and (d) spherical-like erythrocytes. In (b and c) and (e and f) intensity distribution maps of a single RBC, labeled through white dashed line, are reported and processes of detection and tracking of the 3-D positions of foci are highlighted by indicating the positions of foci along the optical axis.

maximization of the Tamura coefficient, ${ }^{38,39}$ while the transverse positions are obtained from the positions of the maximum intensity values in the detected foci planes.

An example of focal spots detection and tracking processes is reported in Fig. 2. Specifically, Figs. 2(a) and 2(d) show extracted erythrocytes, obtained after step (i) by applying the anisotropic diffusion filtering, in the case of isotonic [Fig. 2(a)] and hypotonic [Fig. 2(d)] solutions. The intensity distribution maps along $X-Z$ [Figs. 2(b) and 2(e)] and $Y-Z$ [Figs. 2(c) and 2(f)] planes highlight the detection and 3-D tracking of virtual and real foci of the single erythrocyte, labeled by white dashed line. Notice that only a real focal spot is retrieved for the spherical-like erythrocyte, while both real and virtual foci are detected for the dyscocyte. ${ }^{1}$ In Figs. 2(b) and 2(c), the virtual focus is due to the convex central portion of the $\mathrm{RBC}$, while the positive focal spot is produced by the toroidal external shape. Instead, Figs. 2(e) and 2(f) refer to a sphericallike erythrocyte thus only a positive focal spot is present.

\section{Results and Discussion}

The focal spots stability is investigated by applying the tracking process to focal loci of each RBC of a single QPI reconstruction observed over 5 min. Figures 3(a)-3(e) and Videos 1 and 2 show the results of focal loci tracking for time-lapse sequences of discocytes and spherical-like erythrocytes, respectively. In particular, in Fig. 3(a) (last frame of Video 1), we report the 3-D tracking of both real and virtual foci for discocytes ensemble, while only the real focal plane tracking of the spherical-like erythrocytes ensemble is show in Fig. 3(d) (last frame of Video 2). Positions of focal planes along the optical axis are influenced by the intrinsic fluctuation in RBC thickness maps. In fact, any morphological variation of the whole curvature of the erythrocyte shape alters the wavefront transmitted by RBC thus dynamically varying focal spots position along the optical axis. To prove such a relationship, we calculated the thickness maps for both QPI reconstruction sequences assuming a homogeneous refractive index for the $\mathrm{RBCs}^{24,25}$ In this case, the physical thickness map can be derived from the QPI image by dividing it by a constant value $C=2 \pi \Delta n / \lambda$, where $\Delta n=n_{\text {cell }}-n_{\text {medium }}$ with $n_{\text {cell }}=1.395$ and $n_{\text {medium }}=1.334$, and $\lambda=532 \mathrm{~nm}$ is the laser wavelength. We report an example of two thickness maps in Figs. 3(b) and 3(e), from which we calculate the root-mean-square (RMS) membrane fluctuation map of $\mathrm{RBCs}^{24}$ for discocytes [Fig. 3(c)] and spherical-like erythrocytes [Fig. 3(f)], respectively. Finally, in Fig. 4, we report the time evolution of focal spots [Fig. 4(a)] and the corresponding probability density distributions [Fig. 4(b)], for a single discocyte and a single spherical-like erythrocytes, in the first $10 \mathrm{~s}$ of the experiment. By comparing only the real focal spot trends in time, we reveal that the spherical-like erythrocyte presents a greater focal spot variation than the discocyte, as confirmed by calculating the corresponding standard deviations $\sigma$ [see Fig. 4(b)].

Instead, similar average values $\mu$, compared to those reported in Ref. 1, are retrieved. On the other hand, observing Figs. 3(c) and 3(f), we reveal that spherical-like erythrocytes suffer from greater membrane fluctuations than discocytes, thus a correlation of these two phenomena is evident.

We believe that this aspect can be attributed to (i) the intrinsic shape of spherical RBC that has less contact surface with the substrate and (ii) the hypotonic condition, where the lower osmotic pressure causes greater fluctuation sensitivity.

Nevertheless, we quantitatively evaluate the relation between focal spots variation and membrane fluctuation. Let $\mathbf{Z}_{r}^{(i)}$ and $\mathbf{Z}_{v}^{(i)}$ be the vectors of optical axis positions in time of real and virtual focal spots of the $i$ 'th RBCs in the QPI reconstruction, where $\mathbf{Z}_{v}^{(i)}$ is the null-vector for each spherical-like erythrocyte. For each RBCs, we calculate the standard deviations $\sigma$ of both $\mathbf{Z}_{r}^{(i)}$ and $\mathbf{Z}_{v}^{(i)}$, then we averaged them in order to retrieve the overall focal spots variation of the RBCs' ensemble. In Table 1, we report the calculated values, where the average operator is indicated as $\langle\cdot\rangle$. By calculating the average membrane fluctuation for all RBCs as the spatial average value of the 

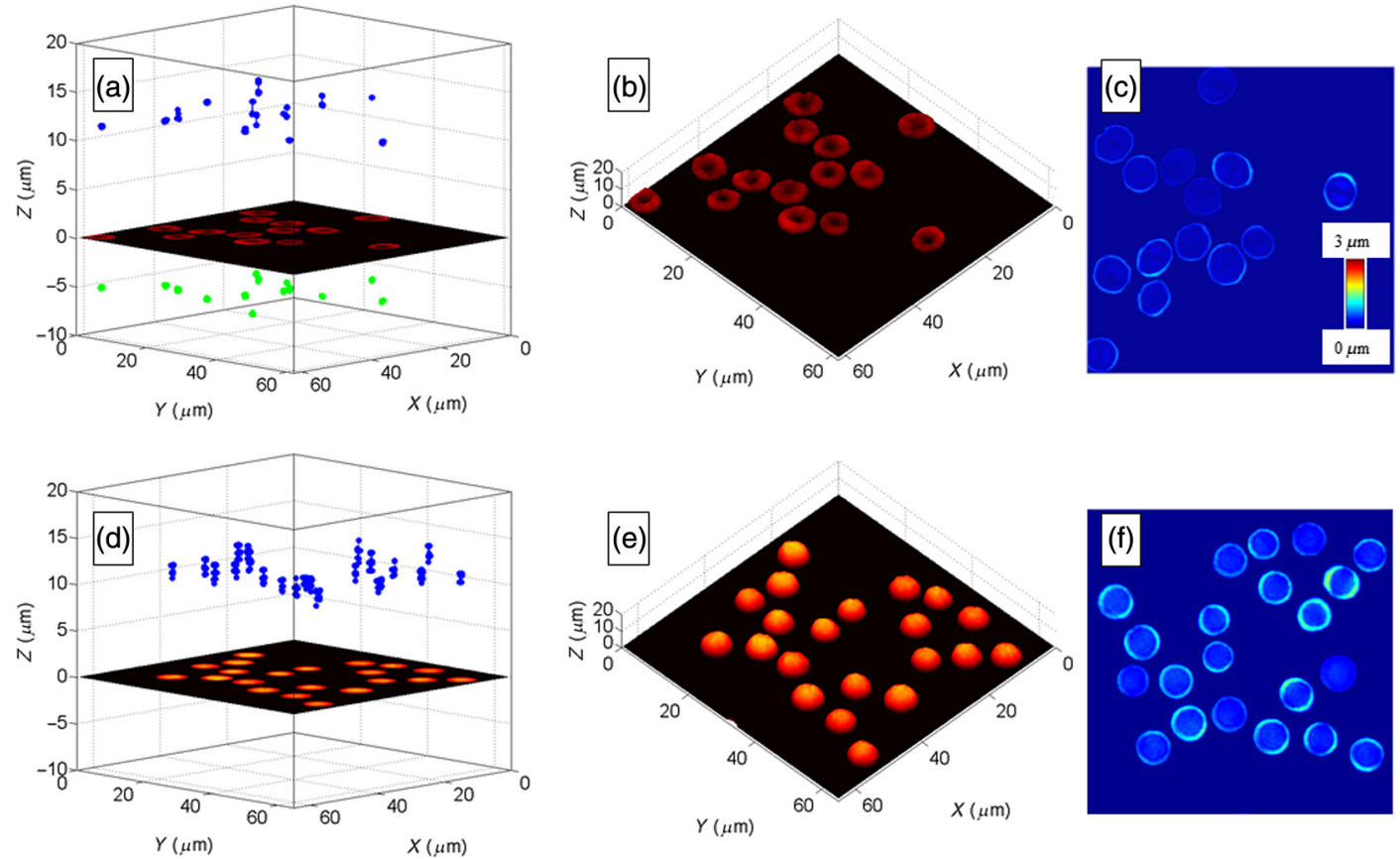

Fig. 3 Results of foci tracking for time-lapse sequences of (a-c) discocytes and (d-f) spherical-like erythrocytes. ( $a$ and $d$ ) report the calculated foci positions, while ( $b$ and $e$ ) and ( $c$ and $f$ ) are the thickness maps and the RMS membrane fluctuation maps, respectively. (Video 1, MOV, 769 KB [URL: http://dx.doi.org/ 10.1117/1.JBO.21.12.121509.1], and Video 2, MOV, 883 KB) [URL: http://dx.doi.org/10.1117/1. JBO.21.12.121509.2], show the temporal tracking of foci and the corresponding thickness map fluctuations, for ( $a$ and $b)$ discocytes and ( $d$ and e) spherical-like erythrocytes, respectively.

RMSs map in Figs. 3(c) and 3(f), we reveal that spherical-like erythrocytes present a two times greater average fluctuation than that of discocytes, (see first row of Table 1), in perfect agreement with the overall focal spots variation.

We also discover that a sort of synchronization occurs among the foci movements. In order to quantify this, we calculate the correlation coefficient (CC) between focal spots vectors for each pair $(i, j)$ of RBCs imaged in the segmented QPI reconstruction, with $I=1, \ldots, N$ and $j=i+1, \ldots, N$, where $N$ is the total number of RBCs, then evaluate the corresponding average values.

In Table 1, the mean value of such coefficients for both discocytes (positive and negative focus, respectively) and spherical-like erythrocytes are reported. These results confirm the correlation in the overall synchronized movements of the focus loci. This evidence in foci synchronization is an important basis for future investigation in exploiting $\mathrm{RBC}$ collective behavior as diagnostic paradigm.
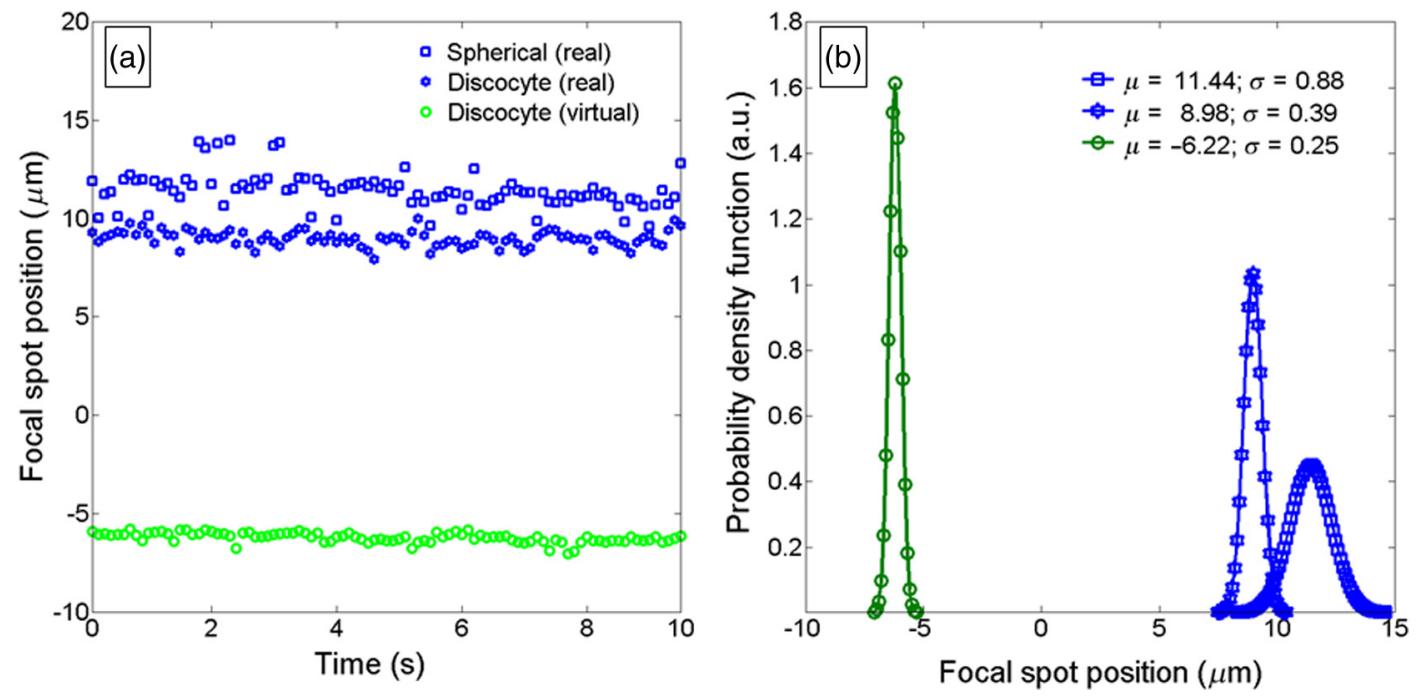

Fig. 4 (a) Time evolution of focal spot position for a single discocyte and a single spherical-like erythrocyte and (b) probability density functions calculated from data in (a). 
Table 1 Average values of the focal lengths standard deviations, mean values of the RBC membrane fluctuations (RMS), and CCs among different focal lengths position variations, for all the RBCs in isotonic solution (i.e., discocytes) and in hypotonic solution (i.e., spherical-like erythrocytes). For discocytes, we have both positive and negative focal lengths, while for spherical-like erythrocytes we only have the positive one.

\begin{tabular}{lcc} 
& Discocytes & Spherical-like erythrocytes \\
\hline$\langle\mathrm{RMS}\rangle(\mu \mathrm{m})$ & 0.68 & 1.42 \\
$\langle\sigma\rangle$ of $Z_{r}(\mu \mathrm{m})$ & 0.58 & 1.18 \\
$\langle\sigma\rangle$ of $Z_{v}(\mu \mathrm{m})$ & 0.23 & $/$ \\
$\langle\mathrm{CC}\rangle$ of $Z_{r}$ & 0.51 & 0.68 \\
$\langle\mathrm{CC}\rangle$ of $Z_{v}$ & 0.54 & $/$ \\
\hline
\end{tabular}

\section{Conclusions}

We report an investigation on RBCs as biophotonic lenses with the aim of characterizing their dynamic behavior. In particular, we have focused the attention on the time-stability of the focal spots of an array produced by the focusing property of RBCs. This is relevant from one side to understand how the living RBCs vary their optical specification for future exploiting as imaging biological optical elements. On the other side, the full characterization of the wavefront properties can be useful for practical purposes in medical diagnostics. In fact, the dynamic stability of foci observed as a result of the present study, allows us to claim that the change in focus for both discocytes and spherical-like erythrocytes is quite small thus avoiding any ambiguity for discriminating different types of RBCs. Therefore, the localization and tracking of their focal spots can be of fundamental importance for the analysis of the healthiness of an RBC through wavefront aberration investigation. In particular, we have studied two different kinds of RBC biolenses, a divergent one (i.e., healthy discocyte) and a convergent one (a spherical-like erythrocytes, i.e., a discocyte in a hypotonic solution). By analyzing an ensemble of each of them, we have studied the membranes' fluctuations and the change in their focal spots positions in time. We quantified these changes through the calculation of different parameters such as the standard deviations of the focal lengths, the spatial average value of RMS map, and the CCs between two RBCs in the ensemble. Moreover, we discovered that the positions of focal spots along the optical axis are in some way synchronized, with an average movement correlation greater than 0.5 in both cases. We hypothesize that a sort of collective movement is present when the RBCs in the ensemble are close to each other at certain characteristic distances and such collective movement influence both membrane fluctuation and foci stability. This last intriguing point needs more in depth investigation for full understanding of how this can occur.

\section{References}

1. L. Miccio et al., "Red blood cell as an adaptive optofluidic microlens," Nat. Commun. 6, 6502 (2015).

2. V. Bianco et al., "Self-propelling bacteria mimic coherent light decorrelation," Opt. Express 23, 9388-9396 (2015).

3. X. Fan and S. H. Yun, "The potential of optofluidic biolasers," Nat. Methods 11, 141-147 (2014).
4. J. Ford, "Red blood cell morphology," Int. J. Lab. Hematol. 35, 351357 (2013).

5. S. H. Cho et al., "Review article: recent advancements in optofluidic flow cytometer," Biomicrofluidics 4(4), 043001 (2010).

6. S. Arpali et al., "High-throughput screening of large volumes of whole blood using structured illumination and fluorescent on-chip imaging," Lab Chip 12, 4968-4971 (2012).

7. H. V. Pham et al., "Real time blood testing using quantitative phase imaging," PLoS One 8, e556761 (2013).

8. P. M. S. Roma et al., "Total three-dimensional imaging of phase objects using defocusing microscopy: application to red blood cells," Appl. Phys. Lett. 104, 251107 (2014).

9. S. Cho et al., "Optical imaging techniques for the study of malaria," Trends Biotechnol. 30(2), 71-79 (2012).

10. M. K. Kim, "Principles and techniques of digital holographic microscopy," SPIE Rev. 1, 018005 (2010).

11. W. Osten et al., "Recent advances in digital holography [Invited]," Appl. Opt. 53, G44-G63 (2014).

12. P. Memmolo et al., "Breakthroughs in photonics 2013: holographic imaging," IEEE Photonics J. 6(2), 701106 (2014).

13. M. Schürmann et al., "Refractive index measurements of single, spherical cells using digital holographic microscopy," Methods Cell Biol. 125, 143-159 (2015).

14. F. Merola et al., "Diagnostic tools for lab-on-chip applications based on coherent imaging microscopy," Proc. IEEE 103(2), 192-204 (2015).

15. P. Memmolo et al., "3D morphometry of red blood cells by digital holography," Cytom. A 85A, 1030-1036 (2014).

16. I. Moon, F. Yi, and B. Rappaz, "Automated tracking of temporal displacements of a red blood cell obtained by time-lapse digital holographic microscopy," Appl. Opt. 55, A86-A94 (2016).

17. F. Yi et al., "Automated segmentation of multiple red blood cells with digital holographic microscopy," J. Biomed. Opt. 18(2), 026006 (2013).

18. I. Moon et al., "Automated quantitative analysis of 3D morphology and mean corpuscular hemoglobin in human red blood cells stored in different periods," Opt. Express 21, 30947-30957 (2013).

19. K. Jaferzadeh and I. Moon, "Quantitative investigation of red blood cell three-dimensional geometric and chemical changes in the storage lesion using digital holographic microscopy," J. Biomed. Opt. 20(11), 111218 (2015).

20. V. Bianco et al., "Imaging adherent cell in microfluidic channel hidden by flowing RBCs as occluding objects by holographic method," Lab Chip 14, 2499-2504 (2014).

21. D. Dannhauser et al., "Optical signature of erythrocytes by light scattering in microfluidic flows," Lab Chip 15, 3278-3285 (2015).

22. K. R. Lee and Y. K. Park, "Quantitative phase imaging unit," Opt. Lett. 39, 3630-3633 (2014).

23. N. T. Shaked, "Quantitative phase microscopy of biological samples using a portable interferometer," Opt. Lett. 37, 2016-2018 (2012).

24. P. Girshovitz and N. T. Shaked, "Fast phase processing in off-axis holography using multiplexing with complex encoding and live-cell fluctuation map calculation in real-time," Opt. Express 23, 8773-8787 (2015).

25. P. Marquet, C. Depeursinge, and P. J. Magistretti, "Review of quantitative phase-digital holographic microscopy: promising novel imaging technique to resolve neuronal network activity and identify cellular biomarkers of psychiatric disorders," Neurophotonics 1(2), 020901 (2014).

26. K. E. Bates and H. Lu, "Optics-Integrated Microfluidic Platforms for Biomolecular Analyses," Biophys. J. 110, 1684-1697 (2016).

27. L. Xue et al., "Single-shot slightly-off-axis interferometry based Hilbert phase microscopy of red blood cells," Biomed. Opt. Express 2, 987-995 (2011).

28. S. Wang et al., "Phase measurements of erythrocytes affected by metal ions with quantitative interferometric microscopy," Opt. Eng. 54, 124105 (2015).

29. F. Merola et al., "Digital holography as a method for $3 \mathrm{D}$ imaging and estimating biovolume of motile cells," Lab Chip 13, 4512-4516 (2013).

30. T. Kim et al., "White-light diffraction tomography of unlabelled live cells," Nat. Photonics 8, 256-263 (2014).

31. G. Popescu et al., "Imaging red blood cell dynamics by quantitative phase microscopy," Blood Cells Mol. Dis. 41(1), 10-16 (2008). 
32. Y. K. Park et al., "Refractive index maps and membrane dynamics of human red blood cells parasitized by Plasmodium falciparum," Proc. Natl. Acad. Sci. U. S. A. 105(37), 13730-13735 (2008).

33. J. Guck et al., "Optical deformability as an inherent cell marker for testing malignant transformation and metastatic competence," Biophys. J. 88, 3689-3698 (2005).

34. D. Holmes et al., "Separation of blood cells with differing deformability using deterministic lateral displacement," Interface Focus 4, 20140011 (2014).

35. P. Memmolo et al., "Recent advances in holographic 3D particle tracking," Adv. Opt. Photonics 7, 713-755 (2015).

36. P. Langehanenberg et al., "Automated three-dimensional tracking of living cells by digital holographic microscopy," J. Biomed. Opt. 14(1), 014018 (2009).

37. P. Memmolo et al., "Identification of bovine sperm head for morphometry analysis in quantitative phase-contrast holographic microscopy," Opt. Express 19(23), 23215-23226 (2011).

38. P. Memmolo et al., "Refocusing criterion via sparsity measurements in digital holography," Opt. Lett. 39, 4719-4722 (2014).

39. P. Memmolo et al., "Automatic focusing in digital holography and its application to stretched holograms," Opt. Lett. 36, 1945-1947 (2011).

Pasquale Memmolo received his $\mathrm{PhD}$ in electronic and telecommunication engineering at the University of Napoli "Federico II" in 2012. Currently, he is a research scientist at National Council of ResearchIstituto di Scienze Applicate e Sistemi Intelligenti "E.Caianiello" (CNRISASI), Pozzuoli, Italy. He has published over 100 scientific contributions among reviewed journal publications, proceedings, and conference papers. His current scientific interests are digital holography, digital image processing, computer vision, 3-D tracking, microscopy, microfluidics, and optofluidics.
Francesco Merola received his MS degree in physics cum laude from the "Federico II" University of Napoli, Italy, in 2006 and his $\mathrm{PhD}$ in fundamental and applied physics at the same university in 2009. In 2007, he joined the CNR-ISASI, Napoli, where he is a researcher. He has published more than 70 papers and proceedings on international journals. His research interests include optical tweezers, digital holographic microscopy, and biomicrofluidics.

Lisa Miccio received her PhD from the University of Florence for the investigation on ferroelectric crystals to realize integrated optical microdevices in 2010. Currently, she is a researcher at the Italian CNR. Her scientific interests are in optics and photonics, in the field of coherent-light imaging, and integration of label-free methods in microfluidic lab-chip devices for biophotonics application. She is the author of 50 original articles, 5 book chapters, and about 40 oral presentations at international conferences.

Martina Mugnano is a graduate in biotechnologies for health with a thesis on 3-D skin model production in vitro and completed the second cycle degree in medical biotechnology. Currently, she is completing her PhD at the University of Naples "Federico II," studying manipulation of cells through light interaction and smart material, under the supervision of Dr. Pietro Ferraro. She is a coauthor of about 10 scientific publications in international journals and conference proceedings.

Pietro Ferraro is currently the director at CNR-ISASI, Pozzuoli, Italy. He has published 12 book chapters, and more than 250 papers in peer-review journals. He edited two books with Springer. He holds 14 patents. Among his current scientific interests are holography, interferometry, microscopy, fabrication of nanostructures, ferroelectric crystals, optical fiber sensors, fiber Bragg gratings, nanomicrofluidics, optofluidics. He is a fellow of SPIE and OSA. 